\title{
Granzyme B Deficiency Protects against Angiotensin II-Induced Cardiac Fibrosis
}

Yue Shen, ${ }^{* \dagger}$ Fang Cheng, ${ }^{\dagger}$ Mehul Sharma, ${ }^{\dagger}$ Yulia Merkulova, ${ }^{\dagger}$ Sheetal A. Raithatha, ${ }^{* \dagger}$ Leigh G. Parkinson, ${ }^{* \dagger}$ Hongyan Zhao, ${ }^{* \dagger}$ Kathryn Westendorf, ${ }^{* \dagger}$ Lubos Bohunek, ${ }^{* \dagger}$ Tatjana Bozin, ${ }^{* \dagger}$ Ivy Hsu, ${ }^{\dagger}$ Lisa S. Ang, ${ }^{\dagger}$ Sarah J. Williams, ${ }^{* \dagger}$ R. Chris Bleackley, John E. Eriksson, Michael A. Seidman, ${ }^{* \mathbb{\pi}}$ Bruce M. McManus, ${ }^{* \dagger}$ and David J. Granville

From the Centre for Heart Lung Innovation,* St. Paul's Hospital, Vancouver, British Columbia, Canada; the Department of Pathology and Laboratory Medicine, ${ }^{\dagger}$ University of British Columbia, Vancouver, British Columbia, Canada; the Department of Biosciences, ${ }^{\ddagger}$ Turku Centre for Biotechnology, Åbo Akademi University, Turku, Finland; the Department of Biochemistry, ${ }^{\S}$ University of Alberta, Edmonton, Alberta, Canada; and the Department of Pathology and Laboratory Medicine, "Providence Health Care, Vancouver, British Columbia, Canada

Accepted for publication September 18, 2015.

Address correspondence to David J. Granville, Ph.D., Centre for Heart Lung Innovation, St. Paul's Hospital, University of British Columbia, 166-1081 Burrard St, Vancouver, BC V6Z 1Y6, Canada. E-mail: david. granville@hli.ubc.ca.

\begin{abstract}
Cardiac fibrosis is observed across diverse etiologies of heart failure. Granzyme B (GzmB) is a serine protease involved in cell-mediated cytotoxicity in conjunction with the pore-forming protein, perforin. Recent evidence suggests that GzmB also contributes to matrix remodeling and fibrosis through an extracellular, perforin-independent process. However, the role of $\mathrm{GzmB}$ in the onset and progression of cardiac fibrosis remains elusive. The present study investigated the role of $\mathrm{GzmB}$ in the pathogenesis of cardiac fibrosis. $\mathrm{GzmB}$ was elevated in fibrotic human hearts and in angiotensin II-induced murine cardiac fibrosis. Genetic deficiency of GzmB reduced angiotensin II-induced cardiac hypertrophy and fibrosis, independently of perforin. GzmB deficiency also reduced microhemorrhage, inflammation, and fibroblast accumulation in vivo. In vitro, GzmB cleaved the endothelial junction protein, vascular endothelial (VE)-cadherin, resulting in the disruption of endothelial barrier function. Together, these results suggest a perforinindependent, extracellular role for GzmB in the pathogenesis of cardiac fibrosis. (Am J Pathol 2016, 186: 87-100; http://dx.doi.org/10.1016/j.ajpath.2015.09.010)
\end{abstract}

Cardiac fibrosis is a common pathological feature of many heart diseases, and a hallmark feature of chronic heart failure. ${ }^{1}$ Heart failure affects more than five million individuals in North America, and costs $>\$ 32$ billion annually in health care services, medications, and lost productivity. ${ }^{2}$ Approximately half of the patients diagnosed with heart failure die within 5 years, often as a direct consequence of reduced cardiac function. ${ }^{3}$ Cardiac fibrosis involves exaggerated deposition and reduced degradation of extracellular matrix (ECM) proteins, which directly affects heart function. ${ }^{4}$ Fibrosis leads to elevated mechanical stiffness, ${ }^{5}$ poor electrical signaling, ${ }^{6}$ and reduced oxygen delivery, ${ }^{7}$ with subsequent reduced cardiac pumping capacity and abnormal heart rhythm. Given this knowledge, new methods or therapeutics that may prevent or treat cardiac fibrosis are needed. However, despite decades of intense research, effective therapies for cardiac fibrosis remain elusive.

Many distinct triggers can contribute to the development of progressive fibrotic disease. Despite having different etiologies, the common trigger for most chronic fibrotic disorders is persistent inflammation, reflected in enhanced production of cytokines and growth factors, up-regulated expression of proteolytic enzymes, and elevated infiltration of inflammatory cells. ${ }^{8}$ Persistent inflammation stimulates fibroblasts to produce an excess of ECM proteins, which ultimately impairs normal myocardial architecture and function. 8,9

Although granzyme B (GzmB) was discovered as both an intracellular and an extracellular serine protease, ${ }^{10}$ until

\footnotetext{
Supported by Canadian Institutes of Health Research (CIHR) grants MOP 332654 (D.J.G.) and MOP 133518 (R.C.B.), the CIHR Integrated and Mentored Pulmonary and Cardiovascular Training [CIHR IMPACT (Integrated and Mentored Pulmonary and Cardiovascular Training)] Fellowship (Y.S.), the Academy of Finland grant (F.C.), Frederick Banting and Charles Best Canada Graduate Scholarships (M.S. and Y.M.), and a MITACS Accelerate Postdoctoral Fellowship (L.G.P.).

Disclosures: D.J.G. is the Founder and Chief Scientific Officer of viDA Therapeutics Inc.
} 
Table 1 The Clinical Characteristics of Study Patients

\begin{tabular}{lllll}
\hline Study group & Case no. & Sex & Age, years & Primary cardiac diagnosis \\
\hline Healthy heart & 1 & Male & 37 & Normal \\
& 2 & Male & 21 & \\
Ischemic heart disease with prior infarction & 3 & Male & 21 & \\
& 4 & Male & 63 & Atherosclerotic coronary artery disease with prior \\
Dilated cardiomyopathy & 5 & Female & 50 & myocardial infarction \\
& 6 & Male & 61 & Idiopathic dilated cardiomyopathy \\
& 7 & Female & 44 & \\
Other diseases & 8 & Male & 26 & \\
& 9 & Male & 61 & Aortic stenosis \\
& 10 & Male & 52 & Active sarcoidosis \\
& 11 & Male & 59 & Healed myocarditis \\
& 12 & Male & 56 & Healed myocarditis \\
\hline
\end{tabular}

recently, this protease was primarily viewed as a mediator of immune cell-mediated apoptosis, through a mechanism involving the membrane-perforating molecule, perforin, which allows GzmB entry into the cytoplasm of the target cell. ${ }^{11}$ Early studies overlooked the potential role of extracellular GzmB, using perforin-deficient $\left(\mathrm{Prfl}^{-1-}\right)$ mice to study the role of granzymes in disease with the assumption that perforin is necessary for granzyme internalization and apoptosis. The major shortcoming of this approach was that it ignored any possibility that granzymes could contribute to disease independent of perforin, through extracellular mechanisms. ${ }^{12}$ Over recent years, the traditional pathophysiological understanding of GzmB has been challenged because of increasing data showing that GzmB accumulates in the extracellular space of inflamed tissue, and is present and retains its activity in body fluids collected from patients with diseases associated with aging and chronic inflammation. ${ }^{10,13}$ In the extracellular milieu, independent of perforin, GzmB cleaves ECM into fragments that are capable of promoting chemotaxis and increased inflammation. ${ }^{14-16}$ GzmB also modulates cytokine activation through its extracellular proteolytic activity and contributes to persistent inflammation. ${ }^{13}$ In addition, GzmB can induce vascular permeability through the proteolytic release of ECM-sequestered vascular endothelial growth factor, and promote immune cell transmigration through cleavage of the basement membrane. ${ }^{17,18}$ Although there is mounting evidence for the involvement of GzmB in cellular processes related to fibrosis, there has been no direct link established between GzmB and cardiac fibrosis.

In the present study, using a well-established mouse model of angiotensin II (Ang II)-induced cardiac fibrosis, ${ }^{19,20}$ we demonstrated that GzmB is up-regulated in the fibrotic heart. GzmB deficiency protected against Ang II-induced cardiac hypertrophy and cardiac fibrosis, whereas perforin deficiency had no protective effect. GzmB directly cleaved VEcadherin, a key endothelial cell-cell junction protein, and contributed to the disruption of endothelial barrier function and an increase in vascular permeability. GzmB deficiency attenuated cardiac microvascular permeability, cardiac inflammation, and fibroblast accumulation in Ang II-infused hearts, to result in less cardiac fibrosis. On the basis of these data, we propose a perforin-independent, extracellular role of $\mathrm{GzmB}$ in the pathogenesis of cardiac fibrosis. Targeting extracellular GzmB could be a potential therapeutic strategy to intervene in the progression of cardiac fibrosis.

\section{Materials and Methods}

\section{Human Cardiac Samples}

Tissue samples from cases of heart failure from different etiologies, including atherosclerotic coronary artery disease $(n=3)$, idiopathic dilated cardiomyopathy $(n=4)$, healed myocarditis $(n=2)$, sarcoidosis $(n=1)$, and aortic stenosis $(n=1)$ with established patterns of fibrosis, were identified and provided by the staff of the Cardiovascular Tissue Registry of St. Paul's Hospital and University of British Columbia (UBC) in accordance with their ethics protocols. The clinical characteristics of the patients are summarized in Table 1. The analysis of human samples in this study was approved by UBC/Providence Health Care Research Ethics Board (H14-01716).

\section{Mice}

Male wild-type (WT; C57BL/6J), GzmB-deficient $\left(G z m b^{-/-}\right.$ with C57BL/6J background), and perforin-deficient (Prfl ${ }^{-1-}$ with $\mathrm{C} 57 \mathrm{BL} / 6 \mathrm{~J}$ background) mice were purchased from The Jackson Laboratory (Bar Harbor, ME) and housed at the Genetic Engineered Models facility at the UBC Centre for Heart Lung Innovation, St. Paul's Hospital. Animals were fed and watered ad libitum and maintained on a 12-hour light/dark cycle. All animal procedures were performed in accordance with the guidelines for animal 
Table 2 Primer List for Real-Time PCR

\begin{tabular}{|c|c|c|c|}
\hline Gene name (protein name) & Forward sequence & Reverse sequence & Gene ID \\
\hline Gzmb (GzmB) & $5^{\prime}$-CCACTCTCGACCCTACATGG-3' & 5'-GGCCCCCAAAGTGACATTTATT-3' & 14939 \\
\hline Col1a1 (collagen I) & $5^{\prime}$-GAGCGGAGAGTACTGGATCG-3' & $5^{\prime}-$ TACTCGAACGGGAATCCATC-3' & 12842 \\
\hline Col3a1 (collagen III) & $5^{\prime}-$ TGGTCCTCAGGGTGTAAAGG-3' & $5^{\prime}$-GTCCAGCATCACCTTTTGGT-3' & 12825 \\
\hline Prf1 (perforin) & 5'-GTACAACTTTAATAGCGACACAGTA-3' & $5^{\prime}$-AGTCAAGGTGGAGTGGAGGT-3' & 18646 \\
\hline Vim (vimentin) & $5^{\prime}-\mathrm{CGTCCACACGCACCTACAG-3^{ \prime }}$ & $5^{\prime}$-GGGGGATGAGGAATAGAGGCT-3' & 22352 \\
\hline S100a4 (FSP-1) & $5^{\prime}$-TCCACAAATACTCAGGCAAAGAG- $3^{\prime}$ & 5'-GCAGCTCCCTGGTCAGTAG-3' & 20198 \\
\hline Il6 (IL-6) & $5^{\prime}-$ TAGTCCTTCCTACCCCAАTTTCC-3' & $5^{\prime}$-TTGGTCCTTAGCCACTCCTTC-3' & 16193 \\
\hline $\operatorname{Tnf}(\mathrm{TNF}-\alpha)$ & 5'-СССТСАСАСТСАGАTСАТСТTСТ-3' & $5^{\prime}$-GCTACGACGTGGGCTACAG- $3^{\prime}$ & 21926 \\
\hline Ctgf (CTGF) & $5^{\prime}$-GAAGGGCAAAAAGTGCATCC-3' & $5^{\prime}$-GACAGTTGTAATGGCAGGCA-3' & 14219 \\
\hline Tgfb1 (TGF- $\beta$ ) & $5^{\prime}$-CTCCCGTGGCTTCTAGTGC-3' & 5'-GCCTTAGTTTGGACAGGATCTG-3' & 21803 \\
\hline Fn1 (fibronectin) & 5'-GATGTCCGAACAGCTATTTACCA-3' & 5'-CCTTGCGACTTCAGCCACT-3' & 14268 \\
\hline
\end{tabular}

CTGF, connective tissue growth factor; FSP-1, fibroblast-specific protein-1; GAPDH, glyceraldehyde-3-phosphate dehydrogenase; GzmB, granzyme B; MMP, matrix metalloproteinase; $\alpha$-SMA, $\alpha$-smooth muscle actin; TGF- $\beta$, transforming growth factor- $\beta$; TNF- $\alpha$, tumor necrosis factor- $\alpha$.

experimentation approved by the Animal Care Committee of UBC (A14-0230).

\section{Ang II-Induced Cardiac Fibrosis}

Cardiac fibrosis was induced by Ang II infusion, as previously described. ${ }^{19}$ Briefly, mice aged 3 to 4 months received either a 4-week Ang II (Sigma-Aldrich, St. Louis, MO) infusion at $1000 \mathrm{ng} /$ minute per kilogram or a saline infusion from a s.c. implanted 1004 model ALZET mini osmotic pump (DURECT Corp., Cupertino, CA). An osmotic pump was filled with the appropriate solution, primed at $37^{\circ} \mathrm{C}$ for 24 hours in saline, and surgically implanted s.c. posterior to the scapula of the mouse. During the implantation procedure, mice were anesthetized with gaseous anesthetic at a flow rate of $1.5 \mathrm{~L} /$ minute of oxygen with $1.5 \%$ to $2.5 \%$ of isoflurane delivered via a Baines system using a calibrated tabletop anesthetic machine, administered from a rodent nose cone. Postsurgical pain control consisted of a s.c. injection of buprenorphine.

\section{Hemodynamic Measurements and Echocardiography}

Mean blood pressure and resting heart rates were measured by tail cuff plethysmography using the CODA blood pressure system (Kent Scientific Corp., Torrington, CT) before treatment and every week during Ang II infusion. Echocardiography was performed under mild isoflurane sedation $(0.75 \%$ to $1.25 \%)$ using a VisualSonics Vevo 2100 High-Resolution Imaging System with a $40-\mathrm{MHz}$ frequency transducer (Fujifilm VisualSonics, Toronto, ON, Canada). Two-dimensional M-mode images of the left ventricle at the papillary muscle level were obtained from the left parasternal short axis view. Calculations were made according to the guidelines of the American Society of Echocardiography. ${ }^{21}$

\section{Tissue Collection and Histology}

After 4 weeks of Ang II infusion, animals were euthanized by carbon dioxide and perfused with $0.9 \%$ saline, followed by $10 \%$ formalin. The hearts were carefully dissected immediately after the perfusion, weighed, and fixed in 10\% formalin for 24 hours. Hearts were transversely divided into sections at midventricle along the short axis (ie, perpendicular to the long axis), and the heart was embedded in paraffin or OCT compound. Sections ( $5 \mu \mathrm{m}$ thick) were stained with hematoxylin and eosin, Masson's trichrome for collagen, and Prussian blue for hemosiderin. Quantitative image analysis will be performed using Image-Pro Plus 6.3 (Media Cybernetics, Rockville, MD) software, as described previously. ${ }^{22}$

\section{Histology, Immunohistochemistry, and} Immunofluorescence

Tissue sections were stained and quantified, as described previously. ${ }^{23}$ Toluidine Blue at $\mathrm{pH} 2.0$ was used to stain mast cells. Immunohistochemistry was performed for GzmB (ab4059; Abcam, Cambridge, MA), vimentin (number 5741; Cell Signaling, Beverly, MA), fibroblast-specific protein-1 (ab27957; Abcam), $\alpha$-smooth muscle actin (ab5694; Abcam), and cleaved caspase 3 (number 9661; Cell Signaling). Immunofluorescence staining was performed for CD45 (number 550539; BD Biosciences, Franklin Lakes, NJ), CD68 (MCA1957; AbD Serotec, Raleigh, NC), CD3 (ab5690; Abcam), CD31 (number 553370; BD Biosciences), and vascular endothelial (VE)-cadherin [ab33168 (Abcam) and 

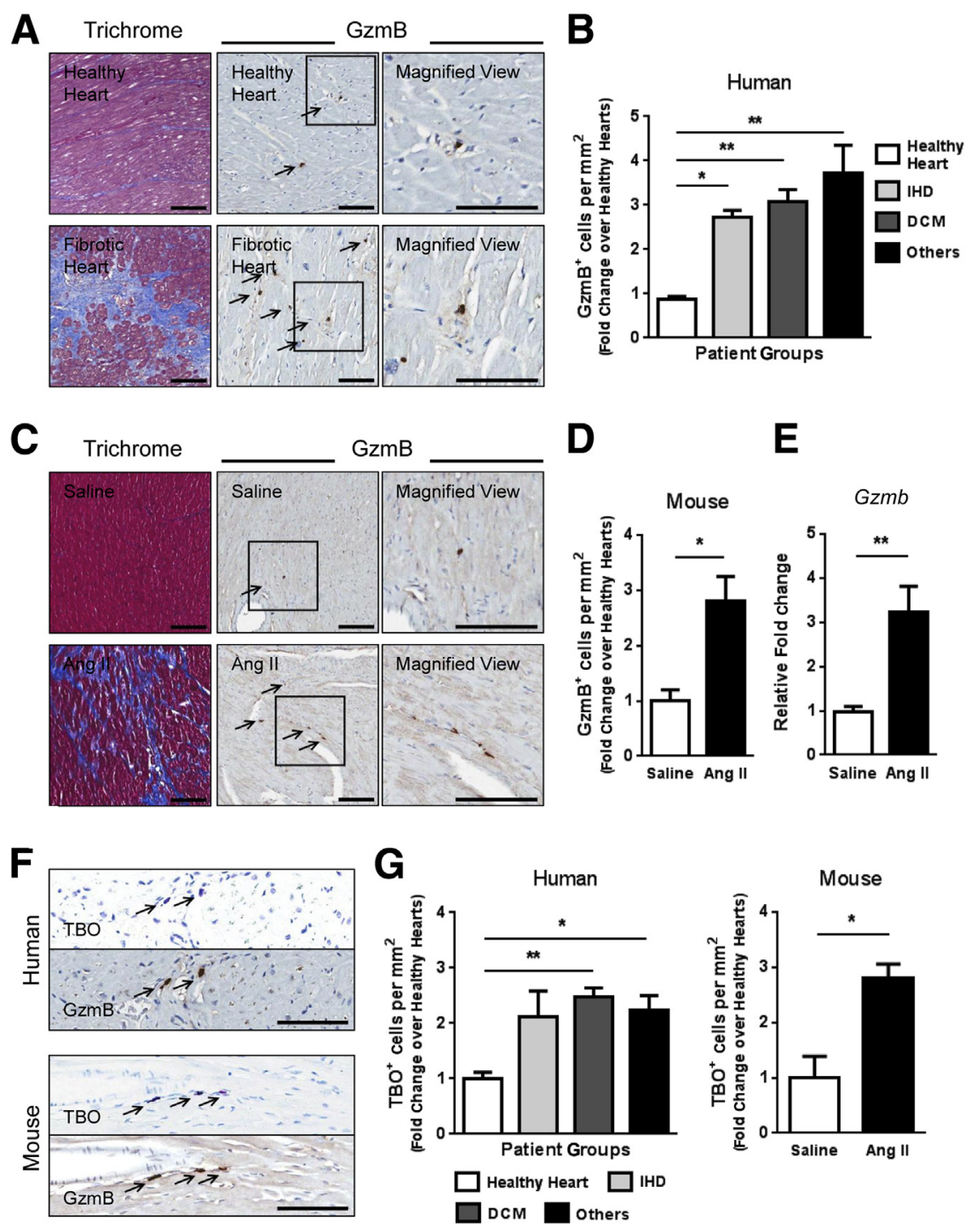

Figure 1 Granzyme B (GzmB) is up-regulated in fibrotic hearts. A: Masson's trichrome staining and GzmB staining of a healthy human heart sample and a sample with cardiac fibrosis. GzmBpositive cells are indicated by arrows. Magnified view (enlarged from boxed area) shows individual GzmB-positive cells. B: Semiquantitative analysis of GzmB-positive cells in the hearts of different patient groups compared with normal healthy hearts. C: Masson's trichrome staining and GzmB staining of cardiac tissue from wild-type (WT) mice treated with saline (top panels) and angiotensin II (Ang II; bottom panels) for 4 weeks. GzmBpositive cells are indicated by arrows. Magnified view (enlarged from boxed area) shows individual GzmB-positive cells. D: Semiquantitative analysis of GzmB-positive cells in WT hearts treated with saline or Ang II for 4 weeks. The number of GzmBpositive cells was significantly increased after 4 weeks of Ang II infusion. E: Real-time PCR analysis of GzmB mRNA expression in WT hearts treated with saline or Ang II for 4 weeks. GzmB expression was up-regulated after 4 weeks of Ang II infusion. F: Direct colocalization of GzmB with mast cells in human and mouse cardiac tissue. Sections were first stained with Toluidine Blue (TB0; $\mathrm{pH} 2)$, and the same sections were stained for GzmB after image capture. GzmB-positive cells and mast cells $\left(\mathrm{TBO}^{+}\right.$cells) are indicated by arrows. G: Semiquantitative analysis of mast cells ( $\mathrm{TBO}^{+}$cells) in the hearts of different patient groups compared with normal healthy hearts and in WT mouse hearts treated with saline or Ang II for 4 weeks. Data represent the means \pm SEM (B, D, E, and $\mathbf{G}) . n=3$ (B and $\mathbf{G}$, normal healthy hearts and IHD group); $n=4(\mathbf{D}$, saline treatment, and $\mathbf{G}, \mathrm{DCM}$ and other groups); $n=6$ (D, Ang II treatment, and G, WT mouse hearts treated with saline); $n=10$ (E, WT hearts treated with saline, and $\mathbf{G}$, WT mouse hearts treated with Ang II); $n=14$ (E, WT hearts treated with Ang II). ${ }^{*} P<0.05,{ }^{*} P<0.01$. Scale bars: 100 $\mu \mathrm{m}(\mathbf{A}, \mathbf{C}$, and $\mathbf{F})$. DCM, dilated cardiomyopathy; IHD, ischemic heart disease with prior infarction.
AF1002 (R\&D Systems, Minneapolis, MN)]. TdT in Situ Apoptosis Detection Kit (4810-30-K; R\&D Systems) was used, and terminal deoxynucleotidyl transferase-mediated dUTP nick-end labeling staining was performed according to the manufacturer's manual. Cardiomyocyte size was determined by measuring cell diameter in sections of the left ventricular myocardium stained with wheat germ agglutinin coupled to Alexa Fluor 633 (W21404; Invitrogen, Burlington, ON, Canada), as described previously. ${ }^{24,25}$ Confocal images were acquired with a Leica AOBS SP2 laser-scanning confocal microscope (Leica, Heidelberg, Germany) and Leica Confocal Software TCS SP2 version 2.61 build 1537. Images were analyzed using Volocity three-dimensional image analysis software version 5.2.1 build 0 (PerkinElmer, Waltham, MA).

\section{Real-Time Quantitative PCR}

Total RNA was extracted from hearts using formalin-fixed, paraffin-embedded Total RNA Isolation Kit (Invitrogen).
cDNA was obtained by reverse transcribing a uniform amount of total RNA using the High Capacity cDNA Reverse Transcription Kit (Applied Biosystems, Burlington, ON, Canada). The transcript levels of the genes of interest were measured by real-time PCR using the SYBR Green PCR mix (Applied Biosystems) in an Applied Biosystems 7300 detection system (Biorad, Mississauga, ON, Canada). The quality of the quantitative PCR run was determined by standard curves and melting curve analysis. The data were normalized to the expression of a cellular housekeeping gene, glyceraldehyde-3-phosphate dehydrogenase. Primers sequences (forward and reverse) used in this study are listed in Table 2.

\section{VE-Cadherin Cleavage Assay}

Mouse VE-cadherin (5 $\mu \mathrm{g}$; 50192-M08H-50; Sino Biological, Beijing, China) was treated with or without $100 \mathrm{nmol} / \mathrm{L}$ recombinant mouse GzmB (G9278; Sigma-Aldrich) and 

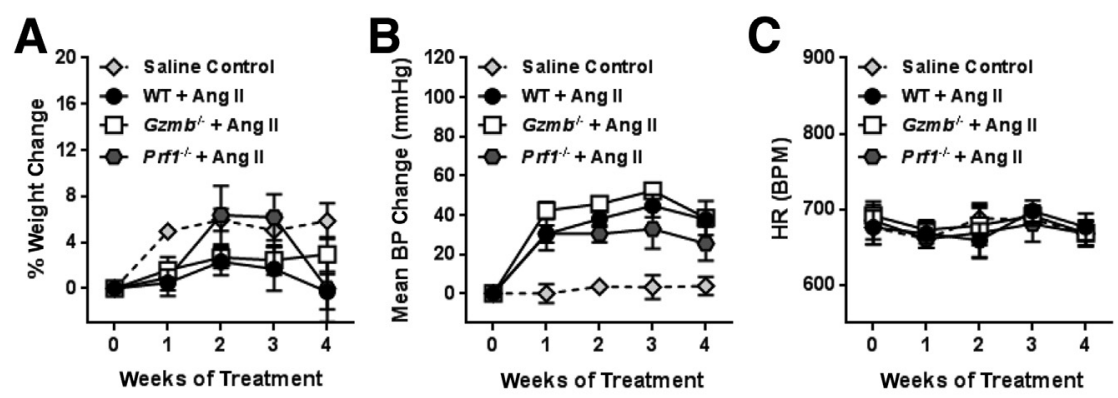

D
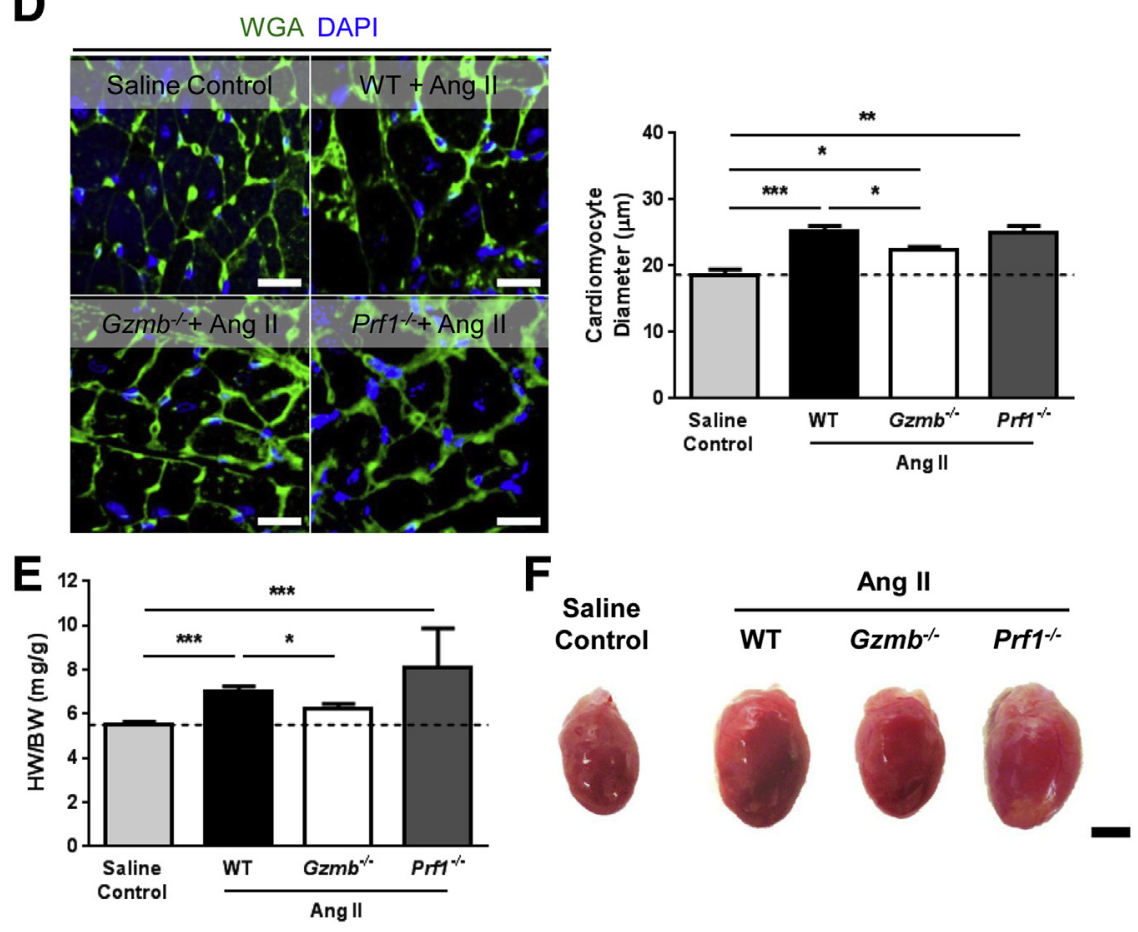

Figure 2 Angiotensin II (Ang II)-induced cardiac hypertrophy in wild-type (WT), granzyme B $\left(\mathrm{Gzmb}^{-/-}\right)$, and perforin-deficient $\left(\mathrm{Prf1} 1^{-/-}\right)$ hearts. Percentage weight (A), mean blood pressure $(B P ; B)$, and heart rate $(H R ; C)$ change after Ang II infusion in WT, Gzmb ${ }^{-/-}$, and Prf1 ${ }^{-/-}$mice for 4 weeks. Saline-infused WT mice were used as saline control to show baseline. Granzyme B (GzmB) or perforin deficiency does not affect the physiological and hemodynamic response to Ang II infusion. D: Quantification of cardiomyocyte diameter in WT mice after 4 weeks of saline treatment (saline control), and in WT, $\mathrm{Gzmb}^{-/-}$ mice, and $\mathrm{Prf1}^{-/-}$mice after 4 weeks of Ang II treatment. Cardiomyocytes were outlined by wheat germ agglutinin (WGA) staining (green). Sections were counterstained with DAPI (blue). E: The heart weight/body weight ratio (HW/BW) in saline control mice, and in WT, $\mathrm{Gzmb}^{-/-}$, and Prf1 ${ }^{-/-}$mice after 4 weeks of Ang II treatment. F: Macroscopic views of representative hearts collected from saline control mice, and from WT, Gzmb ${ }^{-/-}$, and Prf1 ${ }^{-/-}$ mice after 4 weeks of Ang II treatment. Dashed line shows the mean value of saline control group. Data represent the means \pm SEM (A-E). $n=10(\mathbf{A}-\mathbf{C}$, Ang-infused WT mice, D, WT and Gzmb ${ }^{-/-}$mice after Ang treatment, and $\mathbf{E}, \mathrm{Gzmb}^{-/-}$mice after Ang treatment); $n=5$ (A-C, Ang-infused Gzmb ${ }^{-/-}$and $\mathrm{Prf1}^{-/-}$mice and saline-infused WT mice, D, saline control and $P r f 1^{-1-}$ mice after Ang treatment, and E, saline control mice and $\mathrm{Prf1} 1^{-/}$mice after Ang treatment); $n=14$ (E, WT mice after Ang treatment). ${ }^{*} P<0.05,{ }^{* *} P<0.01$, and ${ }^{* * *} P<0.001$. Scale bars: $20 \mu \mathrm{m}$ (D). BPM, beats per minute. incubated overnight at $30^{\circ} \mathrm{C}$ in $\mathrm{GzmB}$ assay buffer $(50 \mathrm{mmol} / \mathrm{L}$ HEPES, pH 7.5, and 0.1\% 3-[(3-cholamidopropyl)-dimethylammonio]-1-propanesulfonate). For inhibition assays, 300 $\mathrm{nmol} / \mathrm{L}$ of the murine serine protease inhibitor Serpin A3N (SA3N; generous gift from Dr. Chris R. Bleackley, University of Alberta, Edmonton, AB, Canada) was preincubated with $100 \mathrm{nmol} / \mathrm{L} \mathrm{GzmB}$ for 1 hour at $30^{\circ} \mathrm{C}$ before the introduction of VE-cadherin. Reactions were stopped with the addition of SDS sample buffer $(0.5 \mathrm{~mol} / \mathrm{L}$ Tris, pH 6.8, 40\% glycerol, 12\% SDS, 20\% dithiothreitol, and $0.25 \%$ bromophenol blue) and boiled for 5 minutes. Antimouse VE-cadherin antibody (AF1002; R\&D Systems) was used for Western blot analysis.

\section{HUVEC Assay}

Human umbilical venous endothelial cells (HUVECs; CC-2517; Lonza, Basel, Switzerland) were analyzed by confocal microscopy after GzmB treatment, as described previously. ${ }^{26}$ Briefly, HUVECs grown on chamber slides (number 154453; Thermo Scientific, Rochester, NY) were incubated with $100 \mathrm{nmol} / \mathrm{L}$ recombinant human GzmB
(Beryllium, Boston, MA) at $37^{\circ} \mathrm{C}$ for 24 hours. For inhibition assays, $100 \mathrm{nmol} / \mathrm{L}$ recombinant human $\mathrm{GzmB}$ was preincubated with $300 \mathrm{nmol} / \mathrm{L} \mathrm{SA} 3 \mathrm{~N}$ at $37^{\circ} \mathrm{C}$ for $45 \mathrm{mi}-$ nutes before being added to the cells. Cells were fixed with $100 \%$ ice-cold methanol for 1 minute at room temperature. Cells were then permeabilized with phosphate-buffered saline containing $0.2 \%(\mathrm{v} / \mathrm{v})$ Triton X-100 (SigmaAldrich, Oakville, ON, Canada) for 10 minutes and blocked in phosphate-buffered saline containing $1 \%(\mathrm{w} / \mathrm{v})$ bovine serum albumin (Sigma-Aldrich, Oakville, ON, Canada) for 30 minutes at room temperature. VE-cadherin antibody (ab33168; Abcam) incubations were performed at room temperature in the above blocking solution for 1 hour at room temperature. DAPI was used for counterstain. Confocal images were acquired with a Leica AOBS SP2 laser-scanning confocal microscope (Leica) and Leica Confocal Software TCS SP2 version 2.61 build 1537.

\section{Transendothelial Electrical Resistance Measurements}

Transendothelial electrical resistance assays were performed as previously described. ${ }^{27}$ Briefly, eight chambered $10 \mathrm{E}+$ 
Table 3 Echocardiographic Assessment on WT, Gzmb ${ }^{-/-}$, and Prf1 ${ }^{-/-}$Mice

\begin{tabular}{|c|c|c|c|c|c|c|}
\hline Measurements & \multicolumn{2}{|l|}{ WT } & \multicolumn{2}{|l|}{$\mathrm{Gzmb}^{-/-}$} & \multicolumn{2}{|l|}{ Prf1 ${ }^{-/-}$} \\
\hline No. of mice & 14 & 14 & 10 & 10 & 5 & 5 \\
\hline $\mathrm{EF}(\%)$ & $56.57 \pm 2.18$ & $52.91 \pm 3.93$ & $57.07 \pm 1.78$ & $51.22 \pm 2.93$ & $57.29 \pm 3.81$ & $52.87 \pm 2.92$ \\
\hline LVEDD (mm) & $3.93 \pm 0.05$ & $3.52 \pm 0.14^{*}$ & $4.10 \pm 0.08$ & $3.91 \pm 0.06^{\dagger}$ & $4.22 \pm 0.05$ & $3.63 \pm 0.05^{\ddagger \ddagger}$ \\
\hline LVEDV $(\mu \mathrm{L})$ & $67.32 \pm 1.90$ & $51.26 \pm 3.50 * *$ & $67.04 \pm 1.73$ & $59.78 \pm 3.42^{\dagger}$ & $69.60 \pm 1.44$ & $54.14 \pm 1.69^{\ddagger \ddagger}$ \\
\hline LVPWd (mm) & $0.54 \pm 0.03$ & $0.57 \pm 0.02$ & $0.52 \pm 0.02$ & $0.51 \pm 0.01^{\dagger}$ & $0.55 \pm 0.04$ & $0.61 \pm 0.06$ \\
\hline LVPWs (mm) & $0.65 \pm 0.02$ & $0.73 \pm 0.03^{*}$ & $0.69 \pm 0.03$ & $0.64 \pm 0.03^{\dagger}$ & $0.70 \pm 0.03$ & $0.77 \pm 0.04$ \\
\hline
\end{tabular}

All results are presented as means \pm SEM.

${ }^{*} P<0.05, * * P<0.01$ versus WT baseline.

${ }^{\dagger} P<0.05$ versus WT Ang II treatment.

${ }^{\ddagger} P<0.05,{ }^{\ddagger} P<0.01$ versus Prf $1^{-/-}$baseline.

Ang II, angiotensin II; EF, ejection fraction, calculated; FS, fractional shortening, calculated; LVEDD, left ventricular end-diastolic dimension; LVEDV, left ventricular end-diastolic volume; LVESD, left ventricular end-systolic dimension; LVESV, left ventricular end-systolic volume; LVPWd, left ventricular diastolic posterior wall thickness; LVPWs, left ventricular systolic posterior wall thickness.

slides were coated with $10 \mathrm{mmol} / \mathrm{L}$ sterile L-cysteine (Applied Biophysics, Troy, NY) for 10 minutes before wells were washed with sterile water. HUVECs (Lonza) were plated at $1 \times 10^{5}$ cells per well in endothelial growth basal medium media supplemented with EGM-2 Singlequot kit (Lonza) for 0.5 hours at room temperature. Slides were then transferred to the Electric Cell-Substrate Impedance Sensing (Applied Biophysics) apparatus, and impedance, resistance, and capacitance were read continuously at multiple frequencies. After 48 hours in vitro, once cells had become confluent, medium with serum and growth factors was removed and replaced with serum-free EBM medium and returned to the apparatus for continuous read. After stabilizing ( 2 hours), $100 \mathrm{nmol} / \mathrm{L}$ recombinant human GzmB (Beryllium) was added. For SA3N inhibition, $100 \mathrm{nmol} / \mathrm{L}$ recombinant human GzmB (Beryllium) was incubated with $300 \mathrm{nmol} / \mathrm{L} \mathrm{SA} 3 \mathrm{~N}$ at $37^{\circ} \mathrm{C}$ for 45 minutes before being added to the cells. Continuous measurements were then made for the following 72 hours. The average impedance and capacitance during a 1-hour period of time at specific time points were used for further data analysis.

\section{Statistical Analysis}

Quantitative values are expressed as means \pm SEM. Statistical analysis was performed using GraphPad Prism version 5.01 (GraphPad Software, San Diego, CA). One-way analysis of variance with post hoc test or $t$-test was used, where appropriate, for group comparison analyses, with $P<0.05$ considered statistically significant.

\section{Results}

\section{GzmB Is Up-Regulated in the Fibrotic Heart}

To investigate the involvement of GzmB in cardiac fibrosis, diseased human cardiac tissue samples with established fibrosis were examined. Few GzmB-positive cells were observed in control healthy heart tissue, whereas GzmB-positive cells were prominent and diffusely present in fibrotic heart tissue (Figure 1A). Quantification revealed a similar expression pattern in all of the examined fibrotic heart samples regardless of differences in etiology, wherein the number of GzmB-positive cells was increased approximately threefold in comparison to normal hearts (Figure 1B). Using a murine model of Ang II-induced cardiac fibrosis, WT mice exhibited extensive cardiac fibrosis accompanied by elevated levels of GzmB (Figure 1C). Quantitative analysis showed that the number of GzmB-positive cells and the expression of GzmB mRNA were both increased approximately threefold in WT heart tissue after 4 weeks of Ang II treatment (Figure 1, D and E). To further investigate the cellular origin of GzmB in the heart, cardiac tissue sections were first stained with Toluidine Blue for mast cells, followed by immunostaining for GzmB. Our results showed that most GzmB-positive cells in the heart were mast cells (Figure 1F), which were also increased in the diseased hearts (Figure 1G). The costaining of GzmB and CD3 showed that some of the CD3-positive cells were GzmB positive. We observed no colocalization of CD68 and GzmB staining (Supplemental Figure S1).

\section{GzmB Deficiency Protects against Ang II-Induced Cardiac Hypertrophy}

The elevated level of serum Ang II is correlated with the development of hypertension and cardiac hypertrophy. ${ }^{28}$ Ang II-infused WT mice exhibited retarded weight gain during the 4-week treatment period, whereas salineinfused WT mice (saline control) continued to gain weight as expected. A retarded weight gain was also observed in Ang II-infused GzmB-deficient $\left(G z m b^{-l-}\right)$ 
A
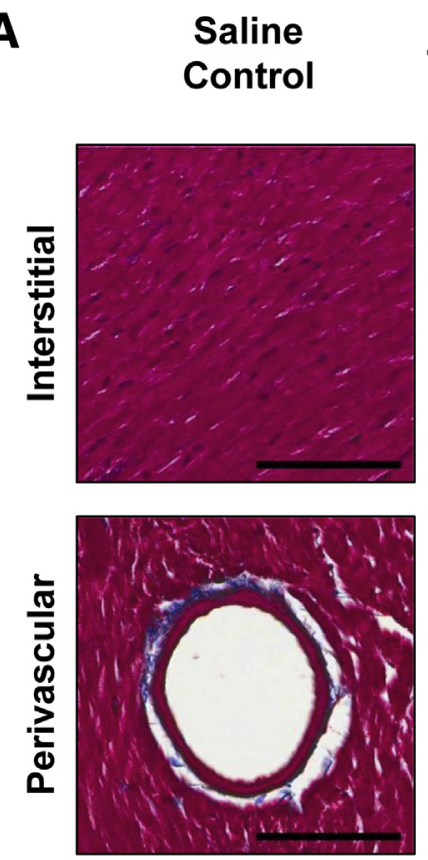

B

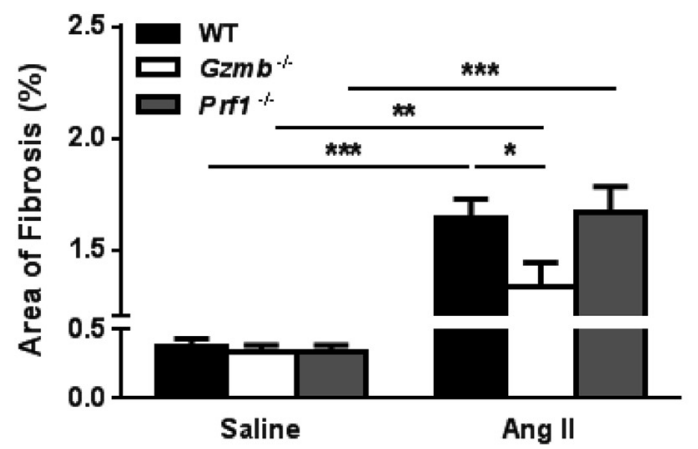

Ang II

WT
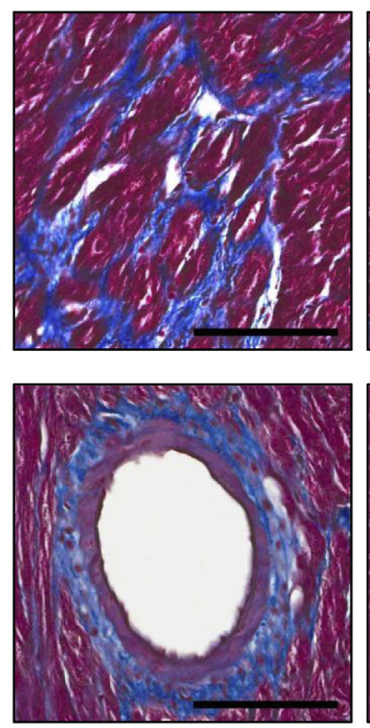

C

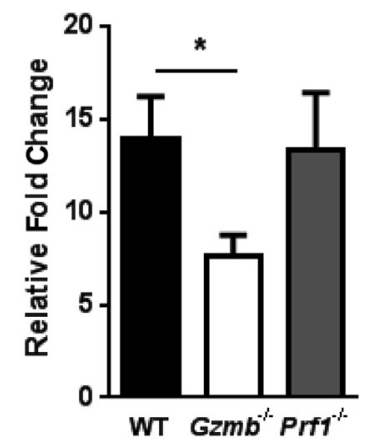

Gzmb-/-
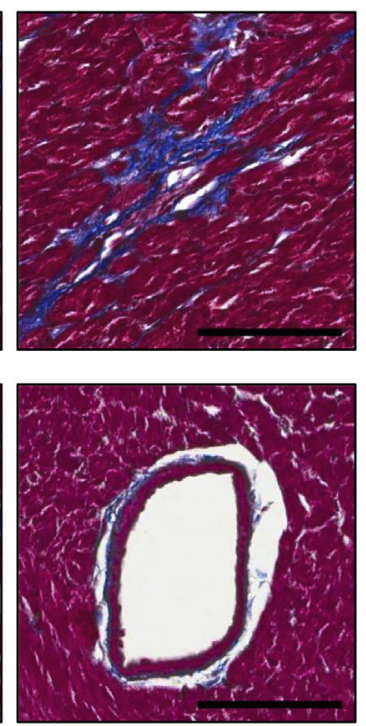

Col1al

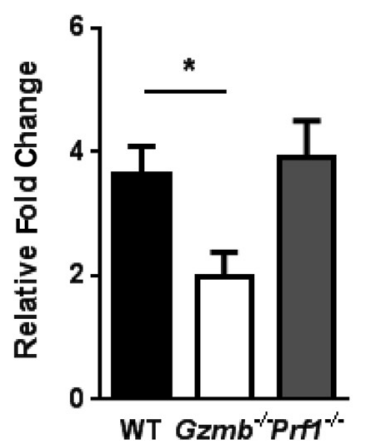

Figure 3 Angiotensin II (Ang II)-induced cardiac fibrosis in wild-type (WT), granzyme B $\left(G z m b^{-/-}\right)$, and perforin-deficient (Prf1 $\left.{ }^{-/-}\right)$hearts. A: Masson's trichrome staining of cardiac tissue from WT mice after 4 weeks of saline treatment (saline control), and from WT, Gzmb ${ }^{-/-}$, and Prf1 $1^{-/-}$mice after 4 weeks of Ang II treatment. B: Quantification of fibrotic area by Masson's trichrome staining in WT, Gzmb ${ }^{-/-}$, and Prf1 ${ }^{-/-}$hearts after 4 weeks of Ang II treatment. C: Real-time PCR analysis of type I and type III collagen (Col) mRNA expression in WT, Gzmb ${ }^{-1-}$, and Prf1 ${ }^{-1-}$ hearts after 4 weeks of Ang II treatment. Data represent the means \pm SEM (B and C). $n=14$ (B and C, WT hearts); $n=10$ (B and C, Gzmb ${ }^{-/-}$hearts); $n=5$ (B and C, Prf1 ${ }^{-/-}$hearts). ${ }^{*} P<0.05$, ${ }^{* *} P<0.01$, and ${ }^{* * *} P<0.001$. Scale bar $=100 \mu \mathrm{m}(\mathbf{A})$.

mice, which was similar to that of WT mice (Figure 2A). Ang II-induced hypertension was observed in WT and $G z m b^{-l-}$ mice, with no difference observed between genotypes (Figure 2B). The heart rates for WT and $G z m b^{-1-}$ mice were similar, and were not affected by Ang II infusion (Figure 2C). The impact of Ang II infusion on cardiac function and anatomy between WT and $G z m b^{-1-}$ mice was further investigated via echocardiography. ${ }^{21}$ Fractional shortening and ejection fraction are the two common measurements of cardiac function. ${ }^{29}$ Both genotypes showed a modest reduction in cardiac function after Ang II treatment (Table 3). Anatomically, although Ang II-infused WT mice had a significantly reduced left ventricle chamber dimension and chamber volume in both diastolic and systolic phases,
Ang II-infused $\mathrm{Gzmb}^{-1-}$ mice were marginally affected. Although the changes of left ventricle posterior wall thickness between baseline and Ang II treatment failed to reach statistical significance, our measurements revealed a trend for an increase of wall thickness in WT mice after Ang II treatment, which was not observed in $G z m b^{-1-}$ mice. Morphologically, cardiomyocyte size was increased in both genotypes after 4 weeks of Ang II infusion; however, the level of Ang II-induced cardiomyocyte hypertrophy in $G z m b^{-1-}$ mice was significantly attenuated compared with WT mice (Figure 2D). These findings were consistent with the measurements of heart weight/body weight ratio and gross observations of hearts harvested from the animals (Figure 2, E and $\mathrm{F}$ ), indicating that GzmB deficiency significantly protected against Ang II-induced cardiac hypertrophy. 
A
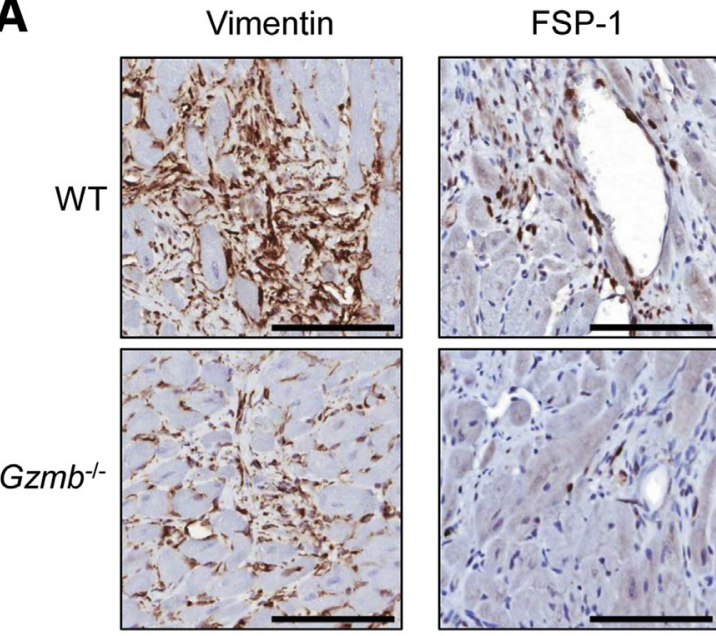

B

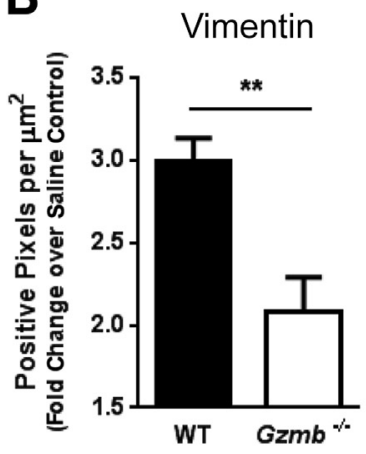

C

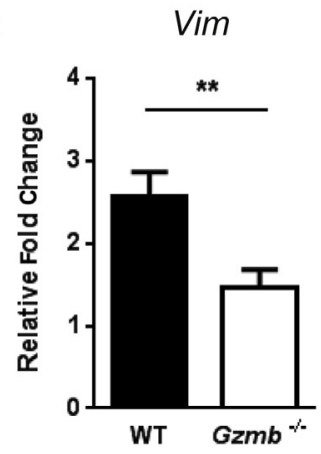

S100a4
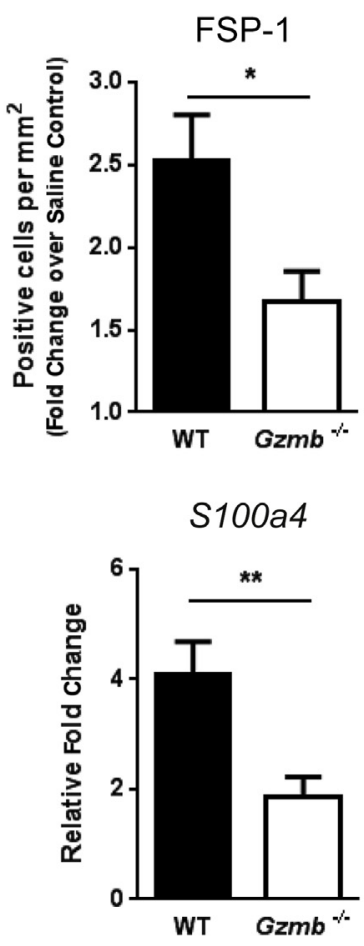

GzmB Deficiency Protects against Ang II-Induced Cardiac Fibrosis

Cardiac fibrosis is commonly found in association with cardiac hypertrophy and failure. ${ }^{30}$ Histological analysis by Masson's trichrome staining revealed excessive collagen deposition in Ang II-infused WT mice hearts, with an interstitial and perivascular distribution. In contrast, fibrosis in Ang II-infused $G z m b^{-1-}$ mice hearts was notably attenuated (Figure 3A). Quantitative analysis of the fibrotic area revealed that, although cardiac fibrosis was prominently developed in both genotypes after 4 weeks of Ang II infusion, the level of fibrosis in $\mathrm{Gzmb}^{-1-}$ mice was significantly lower compared with WT mice (Figure 3B). Real-time quantitative PCR of type I and type III collagen mRNA supported these findings, with
Figure 4 Fibroblast accumulation is attenuated in angiotensin II (Ang II)-infused granzyme B $\left(G_{z m b^{-/-}}\right)$hearts. A: Vimentin (Vim), fibroblastspecific protein-1 (FSP-1), and $\alpha$-smooth muscle actin $(\alpha-$ SMA) staining of cardiac tissue from wildtype (WT) and $\mathrm{Gmb}^{-1-}$ mice after 4 weeks of Ang II treatment. B: Semiquantitative analysis of Vim, FSP- 1 , and $\alpha$-SMA stain in WT and $\mathrm{Gzmb} b^{-/-}$hearts after 4 weeks of Ang II treatment. C: Real-time PCR analysis of Vim, FSP-1 (S100a4), and $\alpha$-SMA (Acta2) mRNA expression in WT and $\mathrm{Gzmb}^{-1-}$ hearts after 4 weeks of Ang II treatment. Data represent the means \pm SEM (B and C). $n=7$ (B, WT hearts); $n=5$ (B, Gzmb ${ }^{-/-}$hearts); $n=14$ (C, WT hearts); $n=10$ (C, Gzmb ${ }^{-1-}$ hearts). ${ }^{*} P<0.05,{ }^{*} P<0.01$. Scale bar $=100 \mu \mathrm{m}(\mathbf{A})$.

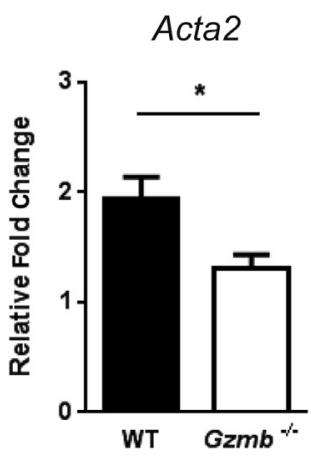

significantly more type I and type III collagen mRNA in WT hearts compared with $G z m b^{-l-}$ hearts (Figure 3C).

Perforin Deficiency Does Not Protect against Ang II-Induced Cardiac Hypertrophy and Fibrosis

The increased level of GzmB mRNA observed after Ang II infusion in WT hearts was also observed in Prfl $^{-1-}$ hearts (Supplemental Figure S2A). However, the same trend was not observed for the levels of perforin mRNA in WT and $G z m b^{-/-}$ hearts after Ang II infusion, having baseline levels similar to the saline-infused controls (Supplemental Figure S2B). Physiological and hemodynamic measurements indicated that $\operatorname{Prfl}^{-1-}$ mice responded similarly to WT mice during the 4-week Ang II treatment period (Figure 2, A-C). The 
A

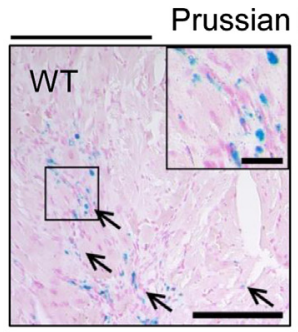

C

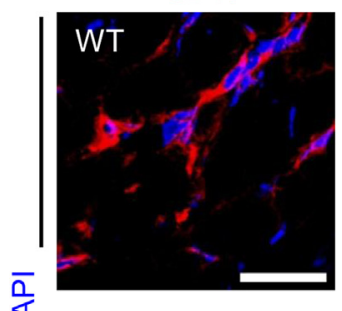

CD45

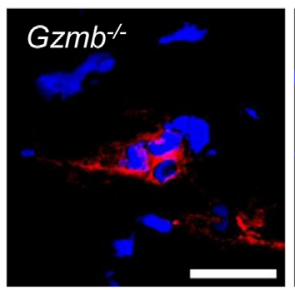

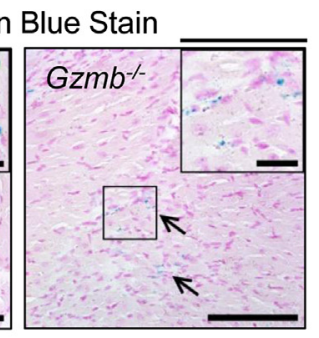

CD68
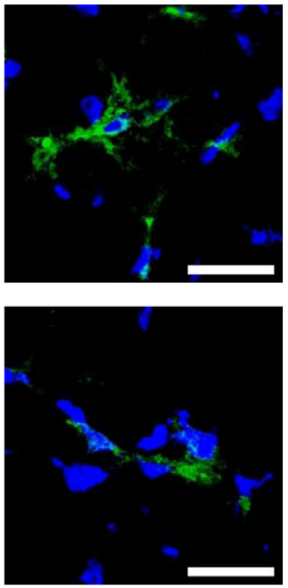

CD68

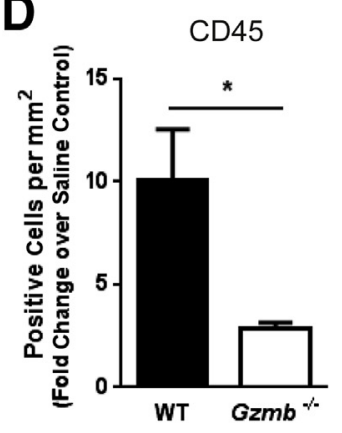

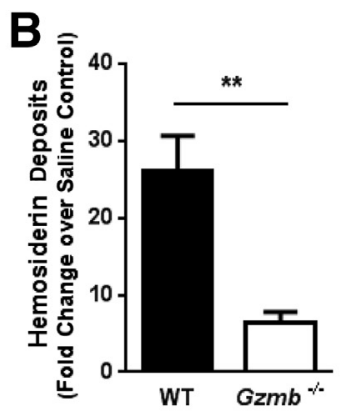
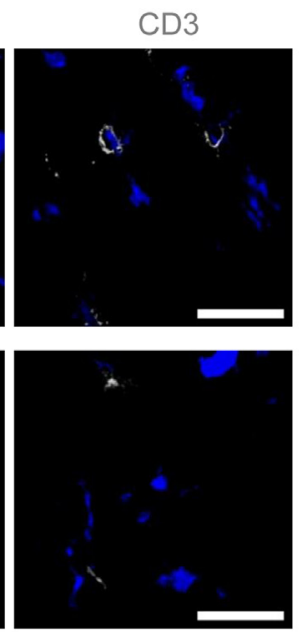
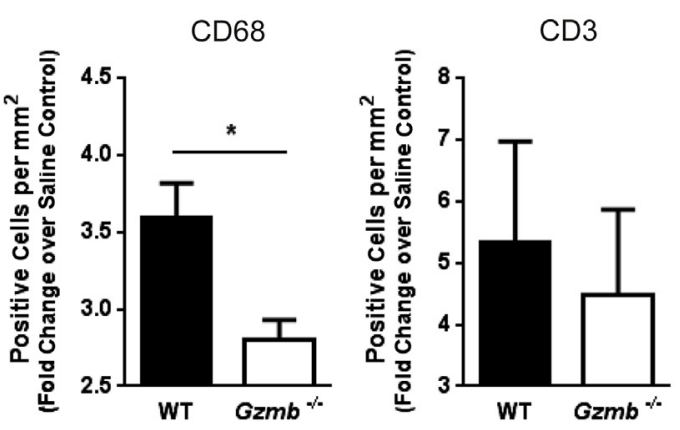

Figure 5 Angiotensin II (Ang II)-induced alteration of cardiac microvascular permeability and inflammation are attenuated in granzyme B $\left(\mathrm{Gzmb}^{-/-}\right)$hearts. A: Prussian blue staining of cardiac tissue from wild-type (WT) and $\mathrm{Gzmb}^{-1-}$ mice after 4 weeks of Ang II treatment. Hemosiderin deposits were stained bright blue and indicated by arrows. Insets: Magnified view of hemosiderin deposits. B: Semiquantitative analysis of hemosiderin deposits in WT and $\mathrm{Gzmb}^{-1-}$ hearts after 4 weeks of Ang II treatment. C: Confocal images of CD45 (red), CD68 (green), and CD3 (gray) staining of cardiac tissue from WT (top panels) and $\mathrm{Gzmb}^{-/-}$mice (bottom panels) after 4 weeks of Ang II treatment. Sections were counterstained with DAPI (blue). D: Semiquantitative analysis of CD45-, CD68-, and CD3-positive cells in WT and $\mathrm{Gzmb}^{-/-}$hearts after 4 weeks of Ang II treatment. E: Real-time PCR analysis of proinflammatory and profibrotic cytokine [Il1b, Il6, tumor necrosis factor (Tnf), connective tissue growth factor (Ctgf), and transforming growth factor- $\beta$ (Tgfb)] mRNA expression in WT and $\mathrm{Gzmb}^{-1-}$ hearts after 4 weeks of Ang II treatment. Data represent the means \pm SEM (B, D, and E). $n=9$ (B, WT); $n=5$ (B and D, Gzmb $\left.{ }^{-1-}\right) ; n=7$ (D, WT); $n=14$ (E, WT); $n=10\left(\mathbf{E}, \mathrm{Gzmb}^{-1-}\right)$. ${ }^{*} P<0.05,{ }^{*} P<0.01$. Scale bars: $100 \mu \mathrm{m}$ (A, main images); $20 \mu \mathrm{m}$ (A, insets); $40 \mu \mathrm{m}(\mathbf{C})$.

\section{$\mathbf{E}$}

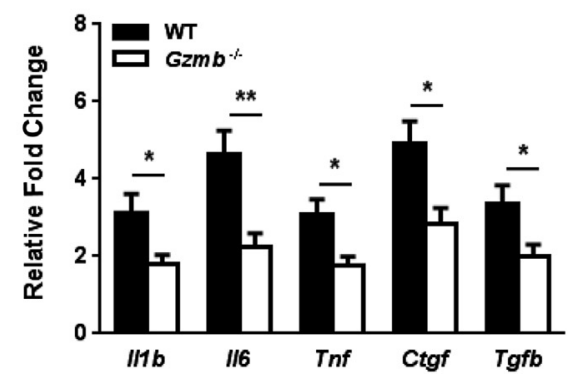

measurements of cardiac function and anatomy of Ang II-infused $\mathrm{Prfl}^{-1-}$ mice revealed that $\mathrm{Prfl}^{-1-}$ mice were not protected and developed cardiac hypertrophy (Table 3 and Figure 2, D-F) and fibrosis (Figure 3A), similar to that observed in WT mice after Ang II treatment. Quantitative analysis of the fibrotic area and real-time PCR for type I and type III collagen mRNA revealed that WT and $\operatorname{Prf1} 1^{-l-}$ hearts had similar levels of increased fibrosis (Figure 3, B and C).

\section{GzmB Deficiency Attenuates Fibroblast Accumulation in Ang II-Induced Cardiac Fibrosis}

Cardiac fibroblasts are the principal determinants of cardiac fibrosis. ${ }^{31}$ Although the fibroblast has been widely studied, no truly definitive cardiac fibroblast marker has been defined. ${ }^{32}$ Therefore, three different fibroblast markers, vimentin, fibroblast-specific protein- 1 , and $\alpha$-smooth muscle actin, were used in the current study to assess the change of fibroblast numbers. Our data revealed a greater number of fibroblast marker-positive cells in WT hearts versus $G z m b^{-l-}$ hearts after 4 weeks of Ang II treatment. The data for all three fibroblast markers were in agreement (Figure 4A). Quantitative analysis of cell staining and realtime PCR measurements of different fibroblast marker transcripts further confirmed that GzmB deficiency attenuated fibroblast accumulation (Figure 4, B and C).

\section{GzmB Deficiency Attenuates Ang II-Induced}

Alteration of Cardiac Microvascular Permeability and Inflammation

To investigate whether GzmB deficiency protects against Ang II-induced cardiac microvascular leakage, Prussian blue iron staining of WT and $G z m b^{-l-}$ hearts after 4-week 
A DAPI
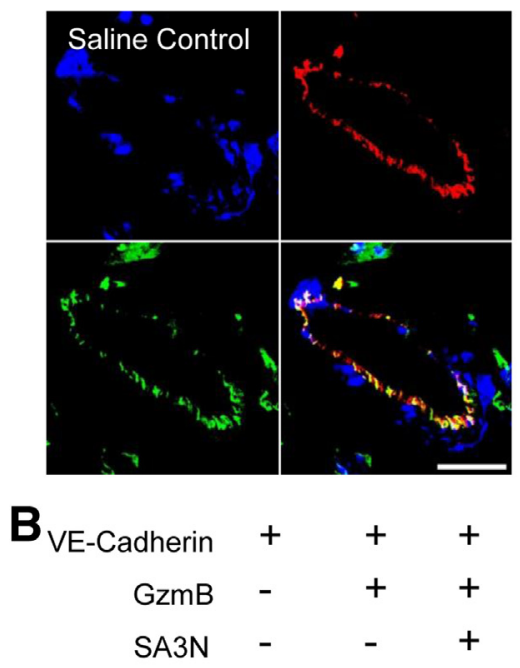

CD31

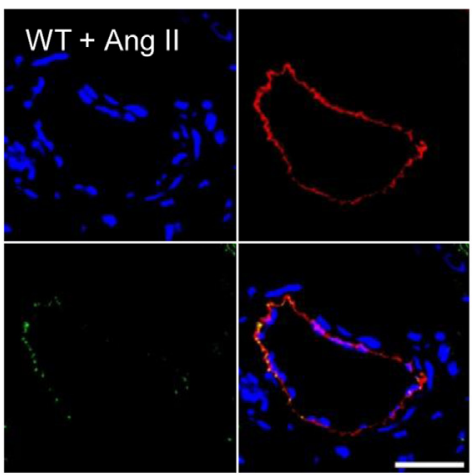

VE-Cadherin

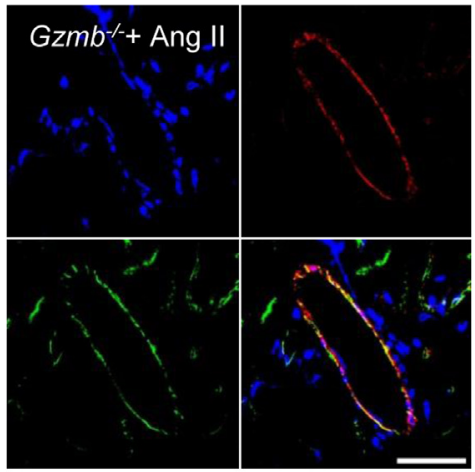

C
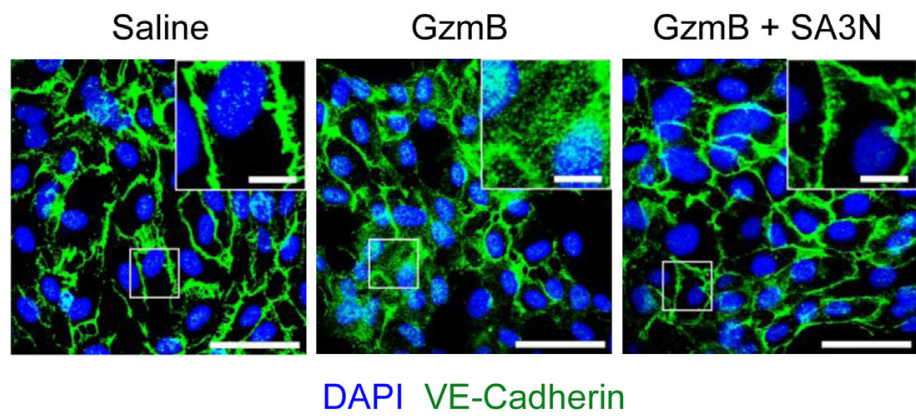

DAPI VE-Cadherin

D

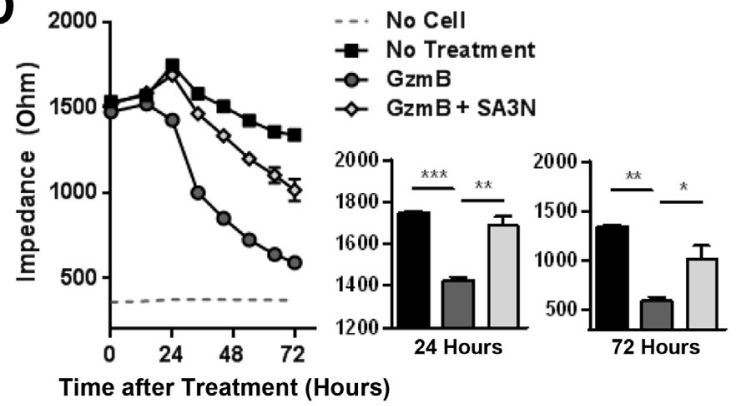

$\mathbf{E}$

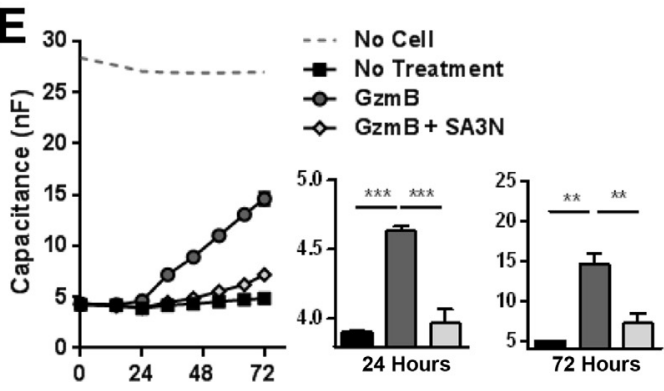

Figure 6 Granzyme B (GzmB) cleaves (VE)-cadherin and contributes to the loss of endothelial barrier function. A: Confocal images of CD31 (red) and vascular endothelial (VE)-cadherin (green) costaining in cardiac tissue from wild-type (WT) mice after 4 weeks of saline treatment and WT and Gzmb ${ }^{-/-}$mice after 4 weeks of angiotensin II (Ang II) treatment. B: Western blot analysis of GzmB-mediated recombinant VE-cadherin cleavage. Arrows indicate VE-cadherin cleavage fragments. The presence of Serpin A3N (SA3N) inhibits GzmB-mediated VE-cadherin cleavage. C: Confocal images of VE-cadherin (green) staining on human umbilical venous endothelial cells (HUVECs) treated with GzmB for 24 hours. Insets: Magnified view of VE-cadherin-stained cell-cell junctions. GzmB disrupts the staining pattern of VEcadherin, which is attenuated by inhibition of GzmB with SA3N. D and E: The effect of GzmB on endothelial barrier function was assessed via transendothelial electrical resistance assays. The change of impedance (D) and capacitance (E) across HUVEC monolayers after no treatment, GzmB treatment, and GzmB + SA3N treatment for 72 hours. An empty well (no cell, gray dashed line) was measured as baseline. The small panels show the impedance (D) and capacitance (E) after no treatment, GzmB treatment, and GzmB + SA3N treatment at 24 and 72 hours after treatment, respectively. Data represent the means \pm SEM $(\mathbf{D}$ and $\mathbf{E}) . n=15$ (D and E, all treatment groups). ${ }^{*} P<0.05,{ }^{* *} P<0.01$, and ${ }^{* * * P}<0.001$. Scale bars: $50 \mu \mathrm{m}$ (A); $100 \mu \mathrm{m}$ (C, main images); $20 \mu \mathrm{m}$ (C, insets).

Ang II treatment was performed to examine the presence of interstitial hemosiderin deposition, an indication of microhemorrhage and increased microvascular permeability. ${ }^{33,34}$ Abundant hemosiderin deposition was present in the fibrotic area of Ang II-infused WT hearts (Figure 5A). In contrast, hemosiderin deposition was noticeably reduced in Ang II-infused $\mathrm{Gzmb}^{-/-}$hearts. Quantitative analysis confirmed that Ang II-induced cardiac microvascular leakage was significantly attenuated in $\mathrm{Gzmb}^{-1-}$ hearts (Figure 5B). Alterations in vascular permeability usually affect leukocyte extravasation. ${ }^{35}$ Immunostaining for three inflammatory cell markers, CD45, CD68, and CD3, revealed a greater number of immune cells in WT hearts versus $G z m b^{-1-}$ hearts after 4 weeks of Ang II treatment (Figure 5, C and D). To further examine the differences in the inflammatory phenotypes observed, real-time PCR of several key cytokines and growth factors that are particularly relevant to fibrosis, ${ }^{36}$ including $I l l b$, $I l 6$, tumor necrosis factor alpha (Tnfa), connective tissue growth factor (Ctgf), and transforming growth factor $\beta(T g f b)$, was performed. For each 


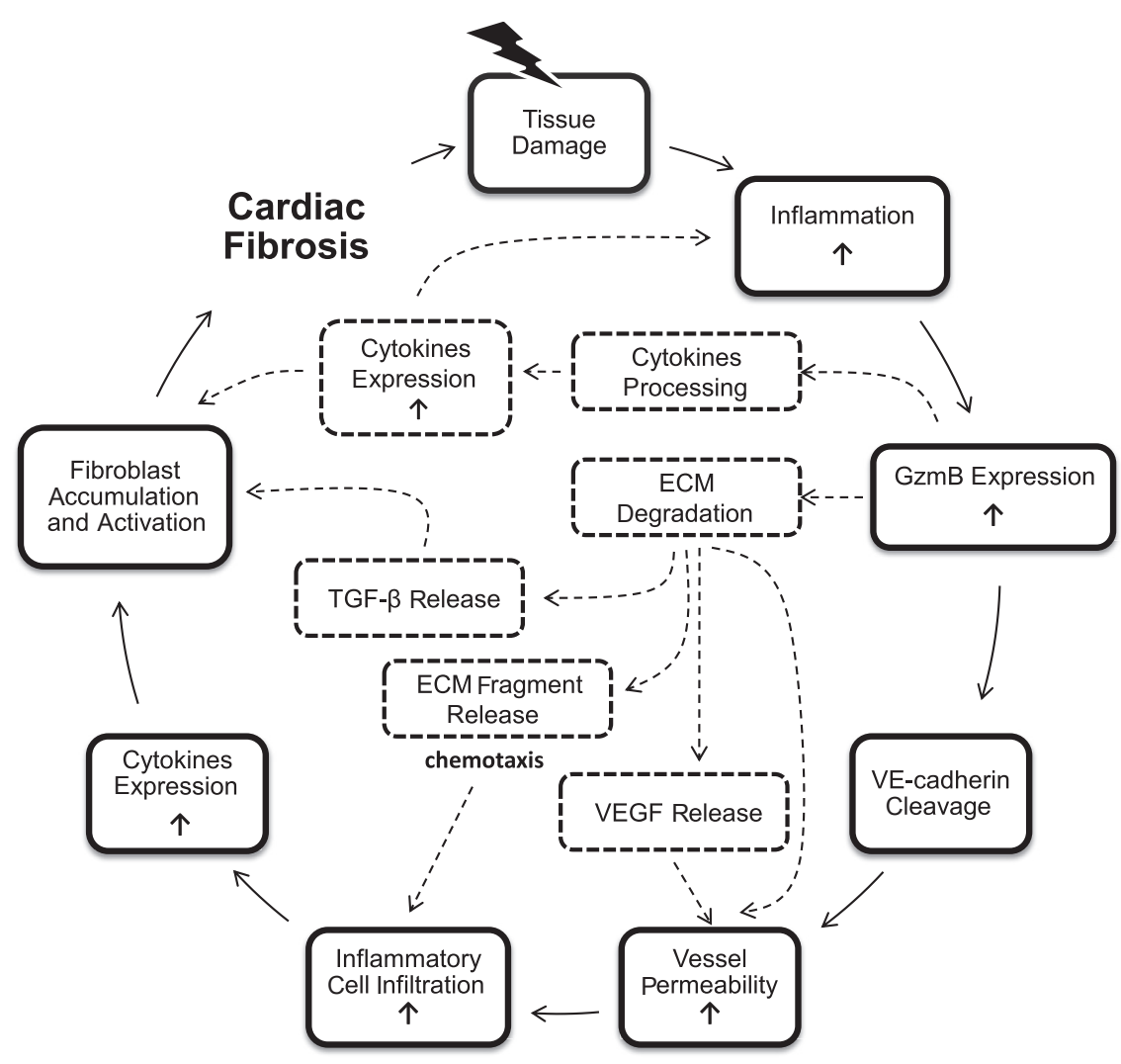

Figure 7 Putative role for perforin-independent, extracellular granzyme B (GzmB)-mediated proteolysis in cardiac fibrosis. On tissue damage, enhanced inflammation leads to an increase in GzmB, which cleaves vascular endothelial (VE)-cadherin. The disruption of VE-cadherin destabilizes cell-cell junctions and causes increased vessel permeability, leading to further inflammatory cell infiltration. In turn, this results in enhanced cytokine expression and promotes a profibrotic phenotype that leads to fibroblast accumulation and fibrosis. The observations from the present study are connected with solid lines. Beyond our current observations involving vascular permeability, other previously reported GzmB-mediated processes, such as extracellular matrix (ECM) degradation, transforming growth factor (TGF)- $\beta$ release, ECM fragment release-induced chemotaxis, vascular endothelial growth factor (VEGF) release, and cytokine processing, may also be involved in the pathogenesis of cardiac fibrosis (dashed lines).

of the previously mentioned factors, the expression was significantly lower in $\mathrm{Gzmb}^{-1-}$ hearts compared with WT hearts after 4 weeks of Ang II treatment (Figure 5E).

\section{GzmB-Mediated VE-Cadherin Cleavage Contributes to the Loss of Endothelial Barrier Function}

VE-cadherin is a key cell junction protein between endothelial cells that is involved in the control of vascular permeability and leukocyte recruitment into tissue. ${ }^{37}$ Costaining of CD31 and VE-cadherin in cardiac tissue showed that the endothelial cell-cell contact was compromised in WT hearts, but remained largely intact in saline control hearts and in $\mathrm{Gzmb}^{-1-}$ hearts after 4 weeks of Ang II treatment (Figure 6A). GzmB directly cleaved VE-cadherin in vitro, resulting in the generation of multiple cleavage fragments (Figure 6B). To assess the contribution of GzmB-mediated VE-cadherin cleavage on disruption of vascular integrity and permeability, HUVECs were treated with GzmB and stained for VE-cadherin. In saline-treated cells, VE-cadherin showed a clear linear staining pattern at cell junctions. Although treatment with GzmB did not affect the cell viability and attachment during the 24-hour treatment period (Supplemental Figure S3), GzmB caused disruption and disorganization of the staining pattern of VEcadherin, which was attenuated by inhibition of GzmB with SA3N (Figure 6C). The effect of GzmB on endothelial barrier function was assessed via transendothelial electrical resistance measurements across endothelial monolayers. GzmB induced a continuous decrease in impedance and a gradual increase in capacitance during the 72-hour treatment period, which was abrogated via cotreatment with SA3N, indicating a GzmBmediated loss of barrier integrity and an increase in permeability (Figure 6, D and E).

\section{Discussion}

The present study showed that GzmB was up-regulated in human fibrotic hearts. Because it is difficult to demonstrate a causative relationship between GzmB and cardiac fibrosis in humans, a reliable animal model was required to manipulate this complex in vivo setting. Continuous systemic infusion of Ang II in a mouse is one of the most widely used animal models for studying the progression of cardiac fibrosis, demonstrating hypertrophy and fibrosis similar to that seen in humans. ${ }^{38,39}$ The results showed that WT mice developed severe cardiac fibrosis accompanied by an increased level of GzmB after 4 weeks of Ang II infusion. Using $G z m b^{-1-}$ mice, we then investigated whether GzmB deficiency affected the Ang II-induced pathological changes observed in the hearts. Although WT and $G z m b^{-1-}$ mice showed similar physiological and hemodynamic responses, Ang II-induced cardiac hypertrophy and fibrosis were significantly attenuated in $G z m b^{-1-}$ mice.

The up-regulation of matrix metalloproteinases (MMPs) is a distinctive feature of cardiac remodeling, particularly when due to chronic infusion of Ang II. ${ }^{40}$ Consistent with previous work, ${ }^{40}$ our measurements also showed a trend for an increase of MMP expression after Ang II treatment. However, there was no significant difference between WT 
and $G z m b^{-1-}$ mice, indicating that GzmB may not play a vital role in the regulation of MMP expression under the current experimental settings (Supplemental Figure S4).

GzmB has been traditionally studied in immune cell-mediated apoptosis, wherein the pore-forming molecule, perforin, plays an essential role. ${ }^{10,11}$ In the past, $\operatorname{Prfl}^{-1-}$ mice were often used to indirectly determine whether granzymes contributed to pathogenesis. On the basis of the assumption that granzymes only function when internalized through a perforin-dependent process, if perforin deficiency had no effect, it was indirectly concluded that GzmB was not involved in the pathophysiology. However, more recent studies have suggested that GzmB may exert significant extracellular and perforin-independent roles in disease. ${ }^{13}$ In support of this concept, the results from the current study demonstrated that the cardioprotective effect of GzmB deficiency was perforin independent and there was no difference in cell apoptosis between WT and $\mathrm{Gzmb}^{-1-}$ hearts at the end of the 4-week Ang II treatment (Supplemental Figure S5).

Cardiac fibroblasts are the driving force in the development of cardiac fibrosis. Cardiac fibroblast accumulation leads to excess ECM deposition and altered cardiac structure and function. ${ }^{41,42}$ However, the lack of a truly specific marker has been a long-standing problem in identifying cardiac fibroblasts. ${ }^{32}$ Vimentin, fibroblast-specific protein-1, and $\alpha$-smooth muscle actin are among the most commonly used fibroblast markers. ${ }^{43}$ Although different markers have different specificities, in the present study, all three markers showed the same trend, wherein the number of cardiac fibroblasts was significantly lower in $G z m b^{-1-}$ hearts compared with WT hearts after 4 weeks of Ang II treatment, indicating that fibroblast accumulation in fibrotic hearts may be, in part, GzmB mediated.

Ang II enhances cardiac microvascular permeability. ${ }^{44}$ Increased cardiac microvascular permeability leads to cardiac microhemorrhage and enhanced local inflammation, which exacerbates cardiac fibrosis. ${ }^{34}$ Previous studies from our group and others showed that GzmB deficiency reduced vascular permeability and inhibited immune cell infiltration in various models of inflammation. ${ }^{17,18}$ Consistent with previous observations, the present study showed that Ang II-induced cardiac microvascular leakage and inflammatory response were attenuated in $\mathrm{Gzmb}^{-/-}$mice but not in $\mathrm{Prfl}^{-/-}$ mice (Supplemental Figure S6), further indicating that GzmB was involved in the regulation of vascular permeability and cardiac inflammation via a perforin-independent mechanism.

VE-cadherin is a key endothelial adhesion molecule that regulates vascular permeability and inflammatory cell recruitment into tissue. ${ }^{37}$ Functional inhibition of VEcadherin leads to increased vascular permeability and accelerated leukocyte extravasation. ${ }^{45}$ GzmB-induced disruption of endothelial cell junctions has previously been reported. ${ }^{46}$ The present study showed that VEcadherin is a proteolytic substrate of GzmB. Furthermore, GzmB treatment of endothelial monolayers impaired cell barrier function and increased permeability. Other studies have shown that GzmB induces vascular permeability via proteolytic release of vascular endothelial growth factor from the $\mathrm{ECM}^{17}$ and promotes leukocyte transmigration via disruption of basement membrane matrix that maintains vessel integrity. ${ }^{18}$ These data support the current in vivo observations, wherein a lower level of vessel permeability, accompanied by reduced leukocyte infiltration, was found in fibrotic $G z \mathrm{mb}^{-/-}$hearts.

Taken together, we propose a perforin-independent, extracellular role for GzmB in the development of cardiac fibrosis (Figure 7). On tissue damage, enhanced inflammation leads to an increase in GzmB, which cleaves VE-cadherin. The disruption of VE-cadherin causes increased vessel permeability and leads to further inflammatory cell infiltration. In turn, this results in enhanced cytokine expression and promotes a profibrotic phenotype that leads to fibroblast accumulation and fibrosis. Beyond the current observation involving vascular permeability, it is possible that GzmB may contribute to other pathophysiological events, including IL processing, release of profibrogenic growth factors, basement membrane degradation, and ECM cleavage fragment-induced chemotaxis, which could exert direct effects on inflammatory cells or directly promote fibroblast proliferation and activation and contribute to the development and progression of cardiac fibrosis. ${ }^{13,18,47,48}$

Untreated WT and $G z m b^{-1-}$ mice have no appreciable phenotypes or differences, suggesting that GzmB primarily functions in an induced manner in response to stimuli, such as tissue injury. This model has not, however, been extensively studied to determine whether age-associated cardiac pathology may be affected by GzmB deficiency. As such, further studies are required to determine the full translational scope of the findings presented.

Protease inhibitor-9 is the only known endogenous inhibitor of human GzmB. ${ }^{49}$ However, this inhibitor has been found to be nonfunctional in the highly oxidative extracellular environment, and, as such, is considered to work intracellularly. ${ }^{50}$ To date, there have been no reports of any extracellular GzmB inhibitors in humans. Consequently, unlike MMPs and other proteases in the extracellular milieu, extracellular GzmB activity may not be inhibited. ${ }^{51}$ As such, excessive extracellular GzmB and its associated proteolytic activities could lead to significant pathophysiologic manifestations, if left unchecked. The current study furthers our understanding of extracellular GzmB in the pathogenesis of cardiac fibrosis, and may ultimately lead to the development of novel therapeutics for preventing or treating cardiac fibrosis by targeting extracellular GzmB.

\section{Acknowledgments}

We thank Meng Wang, Furquan Shaheen, Amrit Samra, Alana Jackson, Stephanie Santacruz, Stephanie Sellers, and Yingjin Wang for technical support and Dr. Chris R. Bleackley 
(University of Alberta, Edmonton, AB, Canada) for providing Serpin A3N.

\section{Supplemental Data}

Supplemental material for this article can be found at http://dx.doi.org/10.1016/j.ajpath.2015.09.010.

\section{References}

1. Berk BC, Fujiwara K, Lehoux S: ECM remodeling in hypertensive heart disease. J Clin Invest 2007, 117:568-575

2. Go AS, Mozaffarian D, Roger VL, Benjamin EJ, Berry JD, Borden WB, et al; American Heart Association Statistics Committee, Stroke Statistics Subcommittee: Heart disease and stroke statistics-2013 update: a report from the American Heart Association. Circulation 2013, 127:e6-e245

3. Yancy CW, Jessup M, Bozkurt B, Butler J, Casey DE Jr, Drazner MH, Fonarow GC, Geraci SA, Horwich T, Januzzi JL, Johnson MR, Kasper EK, Levy WC, Masoudi FA, McBride PE, McMurray JJ, Mitchell JE, Peterson PN, Riegel B, Sam F, Stevenson LW, Tang WH, Tsai EJ, Wilkoff BL: 2013 ACCF/AHA guideline for the management of heart failure: executive summary: a report of the American College of Cardiology Foundation/American Heart Association Task Force on practice guidelines. Circulation 2013, 128:1810-1852

4. Diez J: Mechanisms of cardiac fibrosis in hypertension. J Clin Hypertens (Greenwich) 2007, 9:546-550

5. Chaturvedi RR, Herron T, Simmons R, Shore D, Kumar P, Sethia B, Chua F, Vassiliadis E, Kentish JC: Passive stiffness of myocardium from congenital heart disease and implications for diastole. Circulation 2010, 121:979-988

6. Spach MS, Boineau JP: Microfibrosis produces electrical load variations due to loss of side-to-side cell connections: a major mechanism of structural heart disease arrhythmias. Pacing Clin Electrophysiol 1997, 20:397-413

7. Kai H, Mori T, Tokuda K, Takayama N, Tahara N, Takemiya K, Kudo H, Sugi Y, Fukui D, Yasukawa H, Kuwahara F, Imaizumi T: Pressure overload-induced transient oxidative stress mediates perivascular inflammation and cardiac fibrosis through angiotensin II. Hypertens Res 2006, 29:711-718

8. Wynn TA: Cellular and molecular mechanisms of fibrosis. J Pathol 2008, 214:199-210

9. Wynn TA, Ramalingam TR: Mechanisms of fibrosis: therapeutic translation for fibrotic disease. Nat Med 2012, 18:1028-1040

10. Boivin WA, Cooper DM, Hiebert PR, Granville DJ: Intracellular versus extracellular granzyme B in immunity and disease: challenging the dogma. Lab Invest 2009, 89:1195-1220

11. Jenne DE, Tschopp J: Granzymes, a family of serine proteases released from granules of cytolytic $\mathrm{T}$ lymphocytes upon $\mathrm{T}$ cell receptor stimulation. Immunol Rev 1988, 103:53-71

12. Froelich CJ, Pardo J, Simon MM: Granule-associated serine proteases: granzymes might not just be killer proteases. Trends Immunol 2009, 30:117-123

13. Hiebert PR, Granville DJ: Granzyme B in injury, inflammation, and repair. Trends Mol Med 2012, 18:732-741

14. Buzza MS, Zamurs L, Sun J, Bird CH, Smith AI, Trapani JA, Froelich CJ, Nice EC, Bird PI: Extracellular matrix remodeling by human granzyme B via cleavage of vitronectin, fibronectin, and laminin. J Biol Chem 2005, 280:23549-23558

15. Froelich CJ, Zhang X, Turbov J, Hudig D, Winkler U, Hanna WL: Human granzyme B degrades aggrecan proteoglycan in matrix synthesized by chondrocytes. J Immunol 1993, 151:7161-7171

16. Boivin WA, Shackleford M, Vanden Hoek A, Zhao H, Hackett TL, Knight DA, Granville DJ: Granzyme B cleaves decorin, biglycan and soluble betaglycan, releasing active transforming growth factor-beta1. PLoS One 2012, 7:e33163

17. Hendel A, Hsu I, Granville DJ: Granzyme B releases vascular endothelial growth factor from extracellular matrix and induces vascular permeability. Lab Invest 2014, 94:716-725

18. Prakash MD, Munoz MA, Jain R, Tong PL, Koskinen A, Regner M, Kleifeld O, Ho B, Olson M, Turner SJ, Mrass P, Weninger W, Bird PI: Granzyme B promotes cytotoxic lymphocyte transmigration via basement membrane remodeling. Immunity 2014, 41:960-972

19. Lijnen PJ, Petrov VV, Fagard RH: Induction of cardiac fibrosis by angiotensin II. Methods Find Exp Clin Pharmacol 2000, 22:709-723

20. Hou J, Kato H, Cohen RA, Chobanian AV, Brecher P: Angiotensin II-induced cardiac fibrosis in the rat is increased by chronic inhibition of nitric oxide synthase. J Clin Invest 1995, 96:2469-2477

21. Lang RM, Bierig M, Devereux RB, Flachskampf FA, Foster E, Pellikka PA, Picard MH, Roman MJ, Seward J, Shanewise JS, Solomon SD, Spencer KT, Sutton MS, Stewart WJ; Chamber Quantification Writing Group, American Society of Echocardiography's Guidelines and Standards Committee, European Association of Echocardiography: Recommendations for chamber quantification: a report from the American Society of Echocardiography's Guidelines and Standards Committee and the Chamber Quantification Writing Group, developed in conjunction with the European Association of Echocardiography, a branch of the European Society of Cardiology. J Am Soc Echocardiogr 2005, 18: $1440-1463$

22. Cheung CT, Deisher TA, Luo H, Yanagawa B, Bonigut S, Samra A, Zhao H, Walker EK, McManus BM: Neutralizing anti-4-1BBL treatment improves cardiac function in viral myocarditis. Lab Invest 2007, 87:651-661

23. Hiebert PR, Wu D, Granville DJ: Granzyme B degrades extracellular matrix and contributes to delayed wound closure in apolipoprotein E knockout mice. Cell Death Differ 2013, 20: 1404-1414

24. Ucar A, Gupta SK, Fiedler J, Erikci E, Kardasinski M, Batkai S, Dangwal S, Kumarswamy R, Bang C, Holzmann A, Remke J, Caprio M, Jentzsch C, Engelhardt S, Geisendorf S, Glas C, Hofmann TG, Nessling M, Richter K, Schiffer M, Carrier L, Napp LC, Bauersachs J, Chowdhury K, Thum T: The miRNA212/132 family regulates both cardiac hypertrophy and cardiomyocyte autophagy. Nat Commun 2012, 3:1078

25. Coelho-Filho OR, Shah RV, Mitchell R, Neilan TG, Moreno H Jr, Simonson B, Kwong R, Rosenzweig A, Das S, Jerosch-Herold M: Quantification of cardiomyocyte hypertrophy by cardiac magnetic resonance: implications for early cardiac remodeling. Circulation 2013, 128:1225-1233

26. Ebnet K, Suzuki A, Horikoshi Y, Hirose T, Meyer Zu Brickwedde MK, Ohno S, Vestweber D: The cell polarity protein ASIP/PAR-3 directly associates with junctional adhesion molecule (JAM). EMBO J 2001, 20:3738-3748

27. Szulcek R, Bogaard HJ, van Nieuw Amerongen GP: Electric cellsubstrate impedance sensing for the quantification of endothelial proliferation, barrier function, and motility. J Vis Exp 2014, (85): 51300

28. Diz DI, Baer PG, Nasjletti A: Angiotensin II-induced hypertension in the rat: effects on the plasma concentration, renal excretion, and tissue release of prostaglandins. J Clin Invest 1983, 72:466-477

29. de Simone G, Devereux RB, Roman MJ, Ganau A, Saba PS, Alderman MH, Laragh JH: Assessment of left ventricular function by the midwall fractional shortening/end-systolic stress relation in human hypertension. J Am Coll Cardiol 1994, 23:1444-1451

30. Conrad CH, Brooks WW, Hayes JA, Sen S, Robinson KG, Bing OH: Myocardial fibrosis and stiffness with hypertrophy and heart failure in the spontaneously hypertensive rat. Circulation 1995, 91:161-170

31. Weber KT: Monitoring tissue repair and fibrosis from a distance. Circulation 1997, 96:2488-2492 
32. Souders CA, Bowers SL, Baudino TA: Cardiac fibroblast: the renaissance cell. Circ Res 2009, 105:1164-1176

33. Freidman-Mor Z, Chalon J, Katz JS, Gorstein F, Turndorf H, Orkin LR: Tracheobronchial and pulmonary cytologic changes in shock. J Trauma 1976, 16:58-62

34. Xu Z, Castellino FJ, Ploplis VA: Plasminogen activator inhibitor-1 (PAI-1) is cardioprotective in mice by maintaining microvascular integrity and cardiac architecture. Blood 2010, 115:2038-2047

35. Vestweber D: Relevance of endothelial junctions in leukocyte extravasation and vascular permeability. Ann N Y Acad Sci 2012, 1257:184-192

36. Leask A: Getting to the heart of the matter: new insights into cardiac fibrosis. Circ Res 2015, 116:1269-1276

37. Dejana E, Vestweber D: The role of VE-cadherin in vascular morphogenesis and permeability control. Prog Mol Biol Transl Sci 2013, 116:119-144

38. Harada K, Komuro I, Shiojima I, Hayashi D, Kudoh S, Mizuno T, Kijima K, Matsubara H, Sugaya T, Murakami K, Yazaki Y: Pressure overload induces cardiac hypertrophy in angiotensin II type $1 \mathrm{~A}$ receptor knockout mice. Circulation 1998, 97:1952-1959

39. Li HL, She ZG, Li TB, Wang AB, Yang Q, Wei YS, Wang YG, Liu DP: Overexpression of myofibrillogenesis regulator-1 aggravates cardiac hypertrophy induced by angiotensin II in mice. Hypertension 2007, 49:1399-1408

40. Paolocci N, Tavazzi B, Biondi R, Gluzband YA, Amorini AM, Tocchetti CG, Hejazi M, Caturegli PM, Kajstura J, Lazzarino G, Kass DA Metalloproteinase inhibitor counters high-energy phosphate depletion and AMP deaminase activity enhancing ventricular diastolic compliance in subacute heart failure. J Pharmacol Exp Ther 2006, 317:506-513

41. Weber KT: Fibrosis and hypertensive heart disease. Curr Opin Cardiol 2000, 15:264-272

42. Krenning G, Zeisberg EM, Kalluri R: The origin of fibroblasts and mechanism of cardiac fibrosis. J Cell Physiol 2010, 225:631-637
43. Zeisberg M, Neilson EG: Biomarkers for epithelial-mesenchymal transitions. J Clin Invest 2009, 119:1429-1437

44. Williams B, Baker AQ, Gallacher B, Lodwick D: Angiotensin II increases vascular permeability factor gene expression by human vascular smooth muscle cells. Hypertension 1995, 25:913-917

45. Gotsch U, Borges E, Bosse R, Boggemeyer E, Simon M, Mossmann H, Vestweber D: VE-cadherin antibody accelerates neutrophil recruitment in vivo. J Cell Sci 1997, 110(Pt 5):583-588

46. Pardo J, Wallich R, Ebnet $\mathrm{K}$, Iden $\mathrm{S}$, Zentgraf $\mathrm{H}$, Martin $\mathrm{P}$, Ekiciler A, Prins A, Mullbacher A, Huber M, Simon MM: Granzyme $\mathrm{B}$ is expressed in mouse mast cells in vivo and in vitro and causes delayed cell death independent of perforin. Cell Death Differ 2007, 14:1768-1779

47. Norris DA, Clark RA, Swigart LM, Huff JC, Weston WL, Howell SE: Fibronectin fragment(s) are chemotactic for human peripheral blood monocytes. J Immunol 1982, 129:1612-1618

48. Adair-Kirk TL, Senior RM: Fragments of extracellular matrix as mediators of inflammation. Int J Biochem Cell Biol 2008, 40: $1101-1110$

49. Silverman GA, Bird PI, Carrell RW, Church FC, Coughlin PB Gettins PG, Irving JA, Lomas DA, Luke CJ, Moyer RW, Pemberton PA, Remold-O'Donnell E, Salvesen GS, Travis J, Whisstock JC: The serpins are an expanding superfamily of structurally similar but functionally diverse proteins: evolution, mechanism of inhibition, novel functions, and a revised nomenclature. J Biol Chem 2001, 276:33293-33296

50. Kaiserman D, Bird PI: Control of granzymes by serpins. Cell Death Differ 2010, 17:586-595

51. Kurschus FC, Kleinschmidt M, Fellows E, Dornmair K, Rudolph R, Lilie H, Jenne DE: Killing of target cells by redirected granzyme $\mathrm{B}$ in the absence of perforin. FEBS Lett 2004, 562:87-92 